\title{
PRISMS: A portable multispectral imaging system for remote in situ examination of wall paintings
}

\author{
Haida Liang, ${ }^{*}$ Kafing Keita, Tom Vajzovic \\ School of Science and Technology, Nottingham Trent University, Nottingham NG11 8NS
}

\begin{abstract}
We present a proto-type portable remote multispectral imaging system, PRISMS (Portable Remote Imaging System for Multispectral Scanning), that is light-weight, flexible and without any cumbersome mechanical structure for in situ high resolution colour and spectral imaging of large and inaccessible paintings such as wall paintings. This is the first instrument to be able to image paintings at inaccessible heights in situ from ground level to produce not only high resolution colour images but also multispectral images.
\end{abstract}

Keywords: multispectral imaging, hyperspectral imaging, remote imaging, infrared imaging, pigment identification

\section{INTRODUCTION}

The recording of high resolution spectral images and non-invasive monitoring of wall paintings in grotto sites, tombs and buildings are particularly important since these paintings are extremely vulnerable. The remoteness of some of the sites, the inaccessible height of some of the paintings and the difficulty in controlling the environment they are in, all contribute to their vulnerability. Hence, there is urgency in archiving as precisely as possible the original paintings and in examining their conditions for conservation purposes. Currently, imaging of wall paintings at high resolution requires either scaffolding or some heavy and cumbersome mechanical structure to lift the camera to the upper parts of a wall or ceiling. A number of projects have been devoted to the multispectral and hyperspectral imaging of paintings ${ }^{1,2,3,4,5,6,7,8,9}$, but none has been able to image large and inaccessible paintings remotely at high resolution.

Large format portable cameras such as the MARC for colour imaging ${ }^{10}$ and SIRIS for near infrared imaging ${ }^{11}$ are limited to painting sizes less than $2 \mathrm{~m}$ if sub-millimetre resolutions are required. Traditionally, detailed examinations of wall paintings are only possible with scaffoldings or cherry pickers which are cumbersome, time-consuming, expensive and sometimes dangerous. For example, annual inspections of the Baroque ceiling paintings at the Hampton Court Palace are conducted by lifting a conservator up in a cherry picker. A recent Mellon Foundation funded pilot project to photograph paintings in 40 of the painted caves at the Mogao grottos on the ancient Silk Road, took four years to complete using colour digital photography taken on top of scaffoldings towers ${ }^{12}$. A minimum of 6 people were needed on site. A similar method was used for the imaging of a large modern painting ${ }^{13}$. In the recently completed EU $5^{\text {th }}$ framework VITRA project which was dedicated to the digital three-colour imaging of stained glass in churches ${ }^{14}$, efforts were made to design a flexible system that can operate without the need for scaffolding using a colour digital camera mounted on top of a robotic arm which extended to heights of $15-\mathrm{m}$. While the VITRA project achieved its aims of creating high resolution, colour accurate images of stained glass, the robotic arm system weighing over $300 \mathrm{~kg}$ and over $1-\mathrm{m}$ in diameter and $2.5-\mathrm{m}$ in height when folded up, makes it less than practical for transportation to remote sites and potentially damaging to mosaic floors. There were also problems related to mosaicing of the images because of parallax problems as the camera position was not fixed. A portable, light weight, automatic imaging system installed and operated from ground level is needed even for simple tasks such as colour imaging at sub-millimetre resolution for large paintings and wall paintings in lofty buildings.

In the case of paintings, multispectral imaging was first developed to increase the colour fidelity of the images. In the past 15 years, a number of EU projects have been dedicated to the design and implementation of high colour fidelity, high resolution scanning systems for the recording of museum paintings and other objects of art. In a recently completed EU project CRISATEL, a multi-spectral system was developed from a CCD camera and a filter wheel with a set of 13 interference filters spanning the wavelength range from $400 \mathrm{~nm}$ to $1000 \mathrm{~nm}$. A computer controlled, motorised X-Y

*haida.liang@ntu.ac.uk; phone 44115848 8056; fax 44115848 6636;

O3A: Optics for Arts, Architecture, and Archaeology

edited by Costas Fotakis, Luca Pezzati, Renzo Salimben

Proc. of SPIE Vol. 6618, 661815, (2007) · 0277-786X/07/\$18 - doi: 10.1117/12.726034

Proc. of SPIE Vol. $6618661815-1$ 
scanner moves the camera and the lighting system in front of the painting to take high resolution, multi-spectral images. The individual images collected are automatically mosaiced together using the VIPS/nip software ${ }^{15}$. The system is capable of recovering the spectral reflectance per pixel of a painting with accuracy comparable to a spectrometer for the purpose of colour rendering and pigment identification ${ }^{7}$. Multispectral imaging enables rendering of colour accurate images of paintings under any lighting conditions (unlike a normal tri-colour image which can only capture an accurate colour image under the specific illumination used at the time). In the last three years, hyperspectral imaging using liquid crystal tunable filters (LCTF) has been explored in the $400 \mathrm{~nm}-700 \mathrm{~nm}$ range $^{5,4,8}$ and the $650 \mathrm{~nm}-1040 \mathrm{~nm}$ range ${ }^{6}$. One of the disadvantages of the LCTF is the low blue response. Since the reflectance spectra of pigments in the $400 \mathrm{~nm}$ to $1000 \mathrm{~nm}$ range is fairly smooth, the increased spectral resolution of a hyperspectral system (at the expense of decreased signal to noise) did not provide much additional information. These multi-spectral systems are limited to either scanning a small region at high resolution or a large region at very low resolution.

\section{A PROTO-TYPE REMOTE MULTISPECTRAL IMAGING SYSTEM}

PRISMS consists of a small astronomical telescope, a digital camera and a filter system that operates between 400 and $900 \mathrm{~nm}$. It can be automatically controlled to select filters, focus and take images by a laptop. For imaging at a distance greater than 3.5-m, a Meade EXT-90AT telescope with $90 \mathrm{~mm}$ clear aperture and focal length $1250 \mathrm{~mm}$ is used; and for distances less than 3.5m, a Schneider Componon-S 5.6/150 lens is used. Spatial resolution of the system is between 5 and 25 pixels per millimetre for distances between $1-\mathrm{m}$ and $25-\mathrm{m}$, and sub-millimetre resolutions for distances below $100-\mathrm{m}$. The filter system consists of a filter wheel and a set of 10 interference filters between $400 \mathrm{~nm}$ and $880 \mathrm{~nm}$. The first nine filters have bandwidth of 40nm and are evenly spaced at $50 \mathrm{~nm}$ intervals; the last filter at $880 \mathrm{~nm}$ has a bandwidth of $70 \mathrm{~nm}$. The 14 bit monochrome digital camera, Jenoptik ProgRes $\mathrm{MF}^{\text {cool }}$ with peltier cooling and 5 frames per second download speed (low noise, slow readout mode) has 1360 by 1024 pixels with $6.45 \mu \mathrm{m}$ size pixels. For illumination, a slide projector with a Tungsten Halogen light source $(82 \mathrm{~V} 300 \mathrm{~W})$ is used as a test source. Both the imaging system and the light source stay at ground level during operation. The weight of the camera system is less than $25 \mathrm{~kg}$ and the maximum weight of each component is less than $10-\mathrm{kg}$. The portability of the system means that it can be taken to remote sites to image large paintings in situ at ground level.

The remote multispectral imaging system is shown in Fig. 1 along with the relative spectral responses of the CCD, the transmittance of the telescope and the relative spectral intensity of the light source. Note that the telescope has high throughput in the 400-1700nm range which means PRISMS can be extended to operate as a multispectral/hyperspectral imaging system in the near infrared. The spectral transmittance of the filters is shown in Fig. 2.

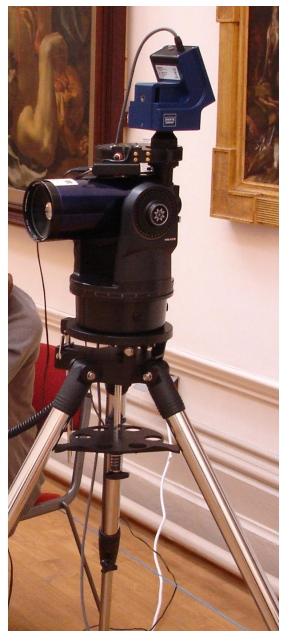

(a)

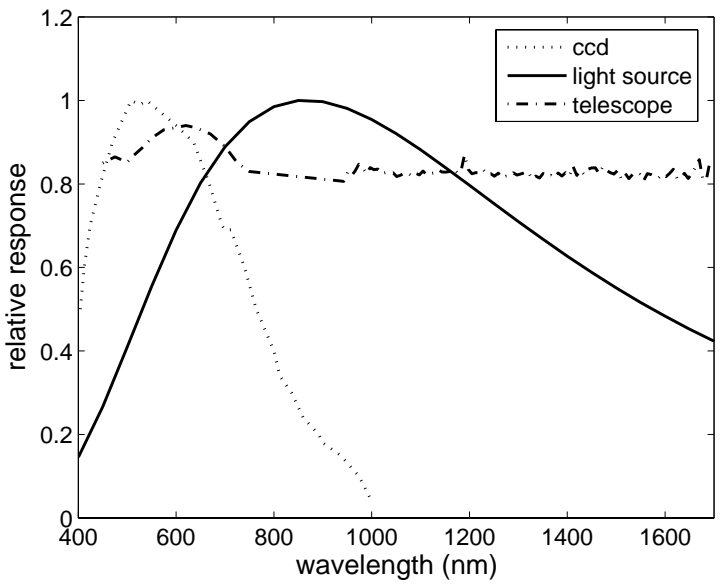

(b)

Fig. 1. a) The proto-type remote multispectral imaging system PRISMS; b) relative spectral response of the CCD, relative spectral transmittance of the telescope and the relative intensity of the light source. 


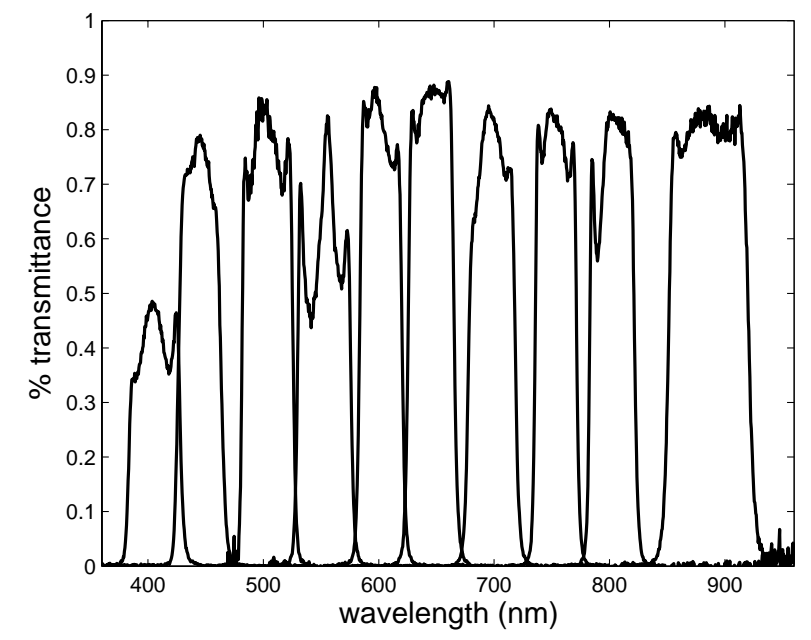

Fig. 2. Transmittance of the filters.

\section{CALIBRATION}

Calibration of a multispectral imaging system involves both the standard CCD calibration procedure and a spectral calibration procedure to adjust the relative spectral response of the filter channels ${ }^{7}$. Integration time for a target is adjusted such that the maximum intensity in an image is about $\sim 10 \%$ below saturation to ensure the best signal to noise. Dark frames taken at the same integration time as the target frames are subtracted from each frame. Flatfield correction for the pixel-to-pixel variation in CCD gain coupled with the inhomogeneity of the illumination can be obtained by imaging a matt white or grey card that is spatially homogeneous and flat. CCD calibration is complete after dark subtraction and flatfield correction. Spectral calibration is achieved by imaging a Labsphere spectralon white standard with known spectral reflectance at the same position as the target.

As an interim solution, a Tungsten-Halogen slide projector is used for illumination as it gives a relatively flat light distribution. However, flatfield correction is still needed. Traditional methods of flatfielding through imaging a matt white board at the same position as the painting can not be used for remote imaging. The calibration has to be performed in the laboratory at various illumination configurations and modelled. Results from using this test source give a valuable handle on the specifications necessary for a remote imaging specific illumination system.

First the stability of the light source needs to be established as it is not a stabilised light source. Figure 3 shows the relative intensity fluctuation measured by a spectrometer with an integration time of $100 \mathrm{~ms}$, when the projector is directly connected to the mains supply. Over the course of 25 minutes, the output intensity from the projector fluctuates within $\sim 1.5 \%$ and over a longer period of $\sim 3$ hours it can change by $\sim 5 \%$. Hence over a relatively short period of 25 minutes, the light source is as good as a stabilised light source designed for spectrometers (e.g. Ocean Optics range of light sources). For longer periods, it would be necessary to monitor and correct for any intensity changes. This can be achieved by imaging a standard white target illuminated by the light source at a fixed position. 

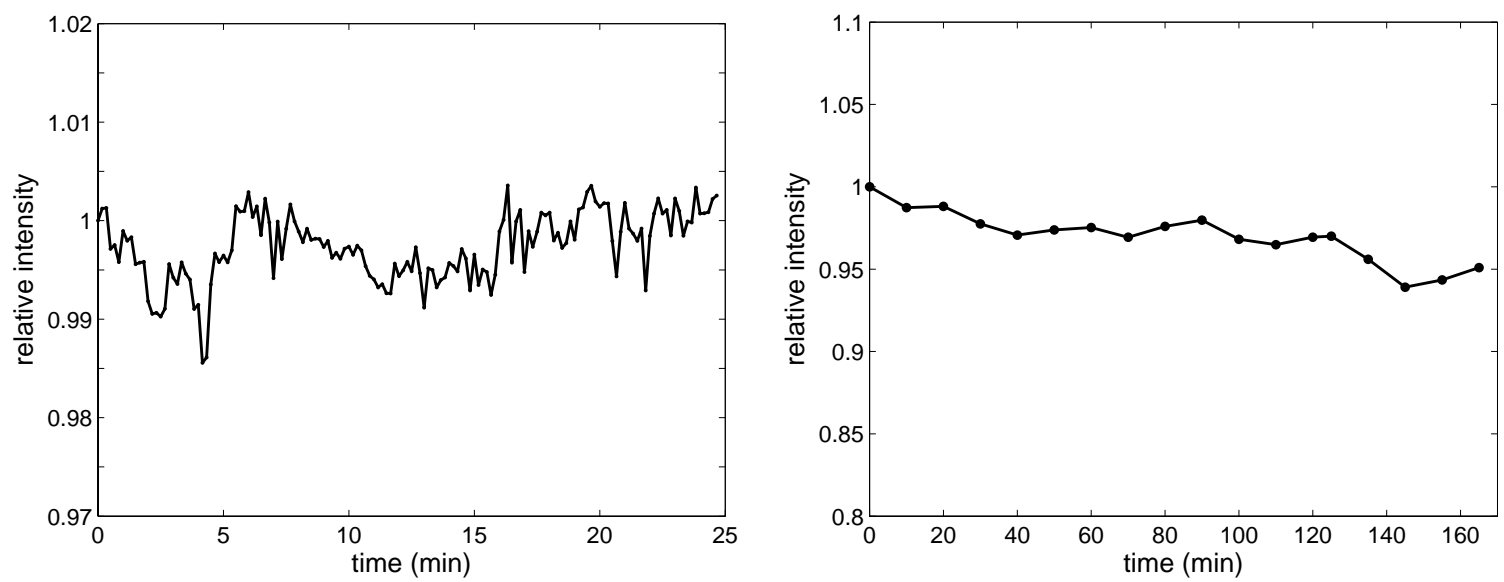

Fig. 3. Stability of the light source measured by a spectrometer (the intensity is averaged over the central $75 \%$ of the measured spectral range) with a $100 \mathrm{~ms}$ integration time: a) over 25 minutes b) over 170 minutes (averaged every 10 minutes).

It is known from basic radiometry that for a Lambertian source, the intensity varies as $I \propto \frac{\cos \theta}{d^{2}}$, where $\theta$ is the angle from the illumination axis and $d$ is the distance between the light source and the target. The intensity of light per pixel incident on the camera is invariant to the distance between the target and the camera. The imaging system was arranged in a retroreflection configuration such that the illumination and imaging axes are aligned. In such a configuration, most of the specular reflection is excluded when the angle of incidence is greater than $\sim 10^{\circ}$. Figure 4 a shows the experimental results of imaging a matt grey card at different distances and Fig. $4 \mathrm{~b}$ shows the results of imaging the grey card at varying angles relative to the illumination/imaging axis. The data show that the simple radiometric relationship for a Lambertian source applies. In addition, the light distribution of a flatfield at different distances and angles stays the same. This is illustrated in Fig. 5 where average profiles along one axis of ratio maps between images of the grey card at two distances and between images at two angles are shown.

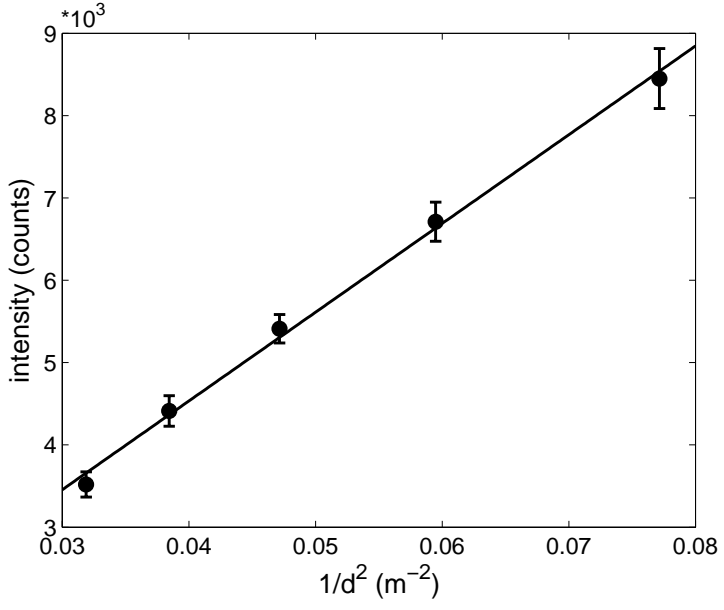

(a)

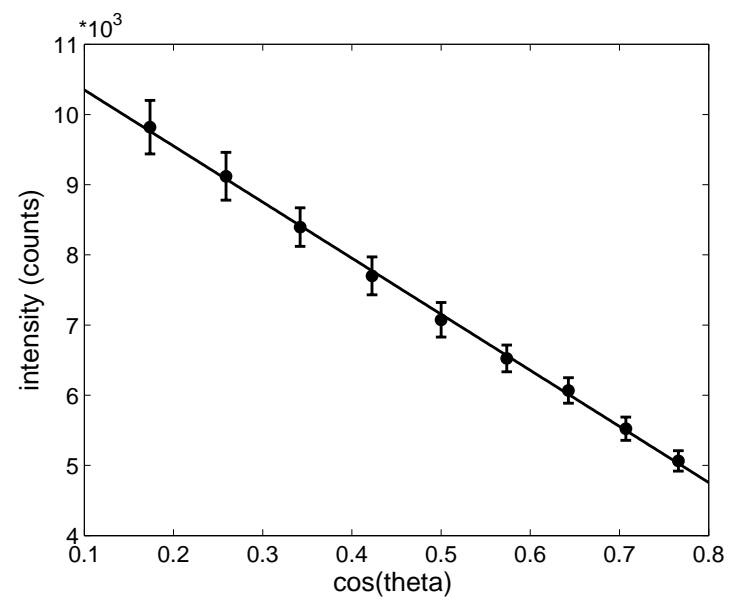

(b)

Fig. 4. a) mean counts of images of a matt grey card illuminated by the projector as a function of $1 / d^{2}$ and $b$ ) as a function of $\cos \theta$. 

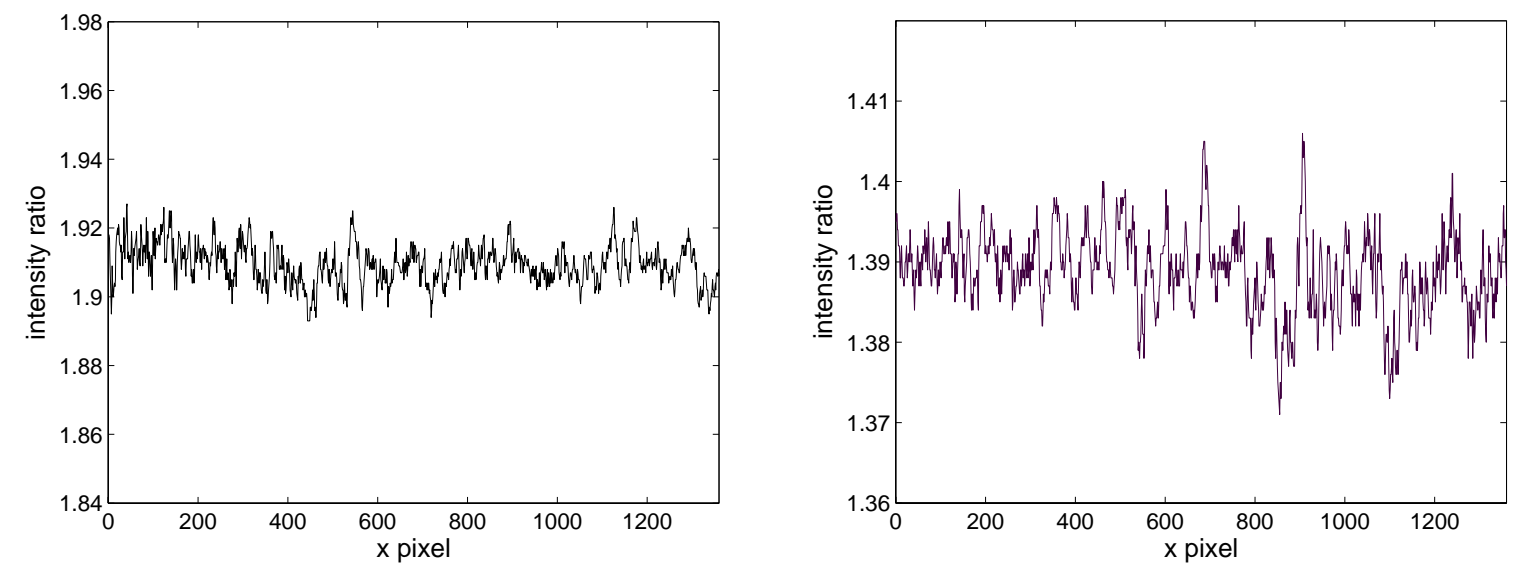

Fig. 5. Left: profile (averaged along the vertical direction) of a ratio map between two images of a smooth grey card imaged at two different distances; Right: average profile of a ratio map between two images of the same grey card at two different angles to the illumination axis.

\section{PRELIMINARY RESULTS \& DISCUSSION}

Two test runs were made: one to test remote imaging by using modelled flatfield based on the calibration curves given in Fig. 4; and another to test multispectral imaging in situ in a gallery during gallery opening hours when natural light from the glass roof is constantly changing.

The first test was to verify that a model predicted image of the grey card corresponds to the actual measured one. The grey card was placed at an arbitrary position and orientation relative to the illumination axis. The image of the grey card was compared with the image predicted from using Fig. 4 and an image of the same grey card taken the previous day at a different but known position. Figure 6 shows the profile averaged along the $\mathrm{x}$-axis and the $\mathrm{y}$-axis of a ratio map between the measured and the predicted flatfield. The predicted flatfield is accurate to $\sim 2 \%$.
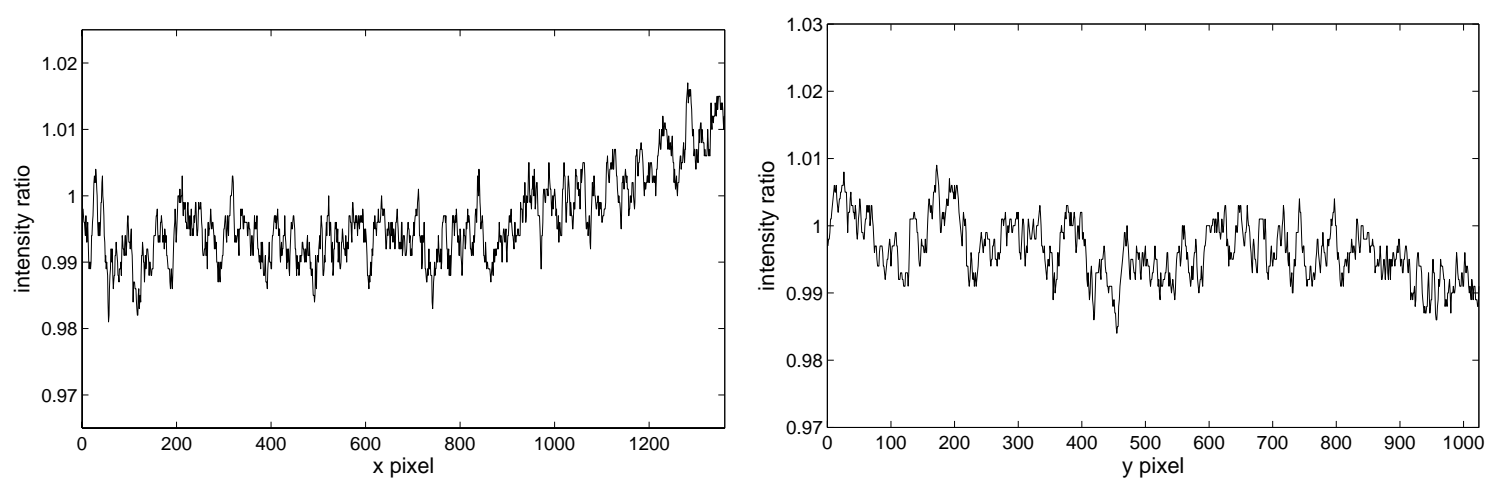

Fig. 6. Left: averaged profile along the y-axis of the ratio map between the predicted and measured flatfields; Right: averaged profile along the $\mathrm{x}$-axis of the same ratio map.

For the second test, the imaging system was brought to the Nottingham Castle Museum (Long Gallery) during opening hours on a sunny but cloudy day. The gallery is lit by a mixture of artificial and natural light which is let through the 
glass roof. As the clouds go past, the lighting level in the gallery changes significantly. The imaging system was set up such that it images a painting 6-m across the gallery, the projector light was placed $0.5-\mathrm{m}$ from the painting/colour chart to increase the efficiency. Once the appropriate integration time was determined per channel, an image was taken of the painting with the projector on and with the projector light blocked, both at the same integration time such that the daylight contribution can be subtracted. The measured spectra of a set of colour patches compared with those measured with a spectrometer are shown in Fig. 7.

The incident light on the painting was $\sim 100$ times the gallery lighting level and it took a total of 10 seconds to image a white standard over the full 10 channels. The integration time was adjusted such that the brightest area of an image is just below saturation ( $90 \%$ of saturation level) and the same signal to noise level is achieved across the channels. Most of the time was spent on imaging the $400 \mathrm{~nm}$ and the $880 \mathrm{~nm}$ channels. The $400 \mathrm{~nm}$ filter has rather low transmittance, which combined with the drop in sensitivity of the CCD and the light intensity in the blue, makes the overall efficiency of the system much lower at $400 \mathrm{~nm}$ than other wavelengths. Similarly, the $880 \mathrm{~nm}$ channel is low in efficiency because of the low sensitivity of the CCD in the infrared. If the light incident on the painting is increased by a factor of 20 , then it will take only 0.5 seconds to image through the full 10 channels. Given that the field of view of the system is $0.5^{\circ}$, it would take 8 hours to image a hemisphere (e.g. in the case of a room or cave that is fully painted ceil to wall). It is known that damage to a painting depends on the total amount of energy exposed. Hence, incident light that is 2,000 times the normal gallery lighting level exposed for 0.5 seconds is equivalent to it being displayed in the gallery for 17 minutes which is insignificant considering that a painting is exposed on average for 8 hours a day in the gallery. In order to achieve such a level of incident illumination, a light source that is $10-\mathrm{m}$ away from the painting would need to be 8,000 times more efficient than the current projector light. While slide projectors are designed to give a uniform illumination they are also designed to have a wide field which is very inefficient as a remote imaging source. If the light is focused into $1^{\circ}$ (the field of view of PRISMS is $0.5^{\circ}$ ), the efficiency of the illumination system would be increased by a factor of 2000. Hence we need $\sim 4$ times as much light output from the bulb. We are in the process of developing an illumination system specific for remote imaging.

Another advantage of using an efficient light source is that the integration time is reduced and hence the effect of blurring due to vibration is also reduced. Galleries and historic homes often use wooden floor which vibrates much more strongly than stone or concrete floors when visitors walk past the equipment.

Figure 8 shows a practical application of remote imaging for inspecting the ceilings of the Queen's staircase at Hampton court palace. This kind of inspection is normally conducted with a cherry picker lifting a conservator up to the ceiling.

\section{CONCLUSIONS \& FUTURE DEVELOPMENT}

We intend to further develop the proto-type by replacing the current illumination system with a more efficient and higher power system specific for remote imaging and by adding an extended NIR hyperspectral system $(0.9-1.7 \mu \mathrm{m})$. The main objectives of the final system is to record high resolution multispectral/hyperspectral images $(0.4 \mu \mathrm{m}-1.7 \mu \mathrm{m})$ from ground level of paintings at distances up to at least $25-\mathrm{m}$, to identify pigments using the spectral reflectance deduced from multispectral/hyperspectral images, and to create a seamlessly mosaiced colour image of a wall painting. The system can be used both qualitatively to assist conservators in examining the conditions of wall paintings and any other large paintings with easy and quantitatively in identifying pigments spectrally and monitoring spectral/colour changes. Compared to colour laser scanning, this system has less emphasis on 3D modelling, but has the advantage of high spectral resolution imaging and is less expensive. 

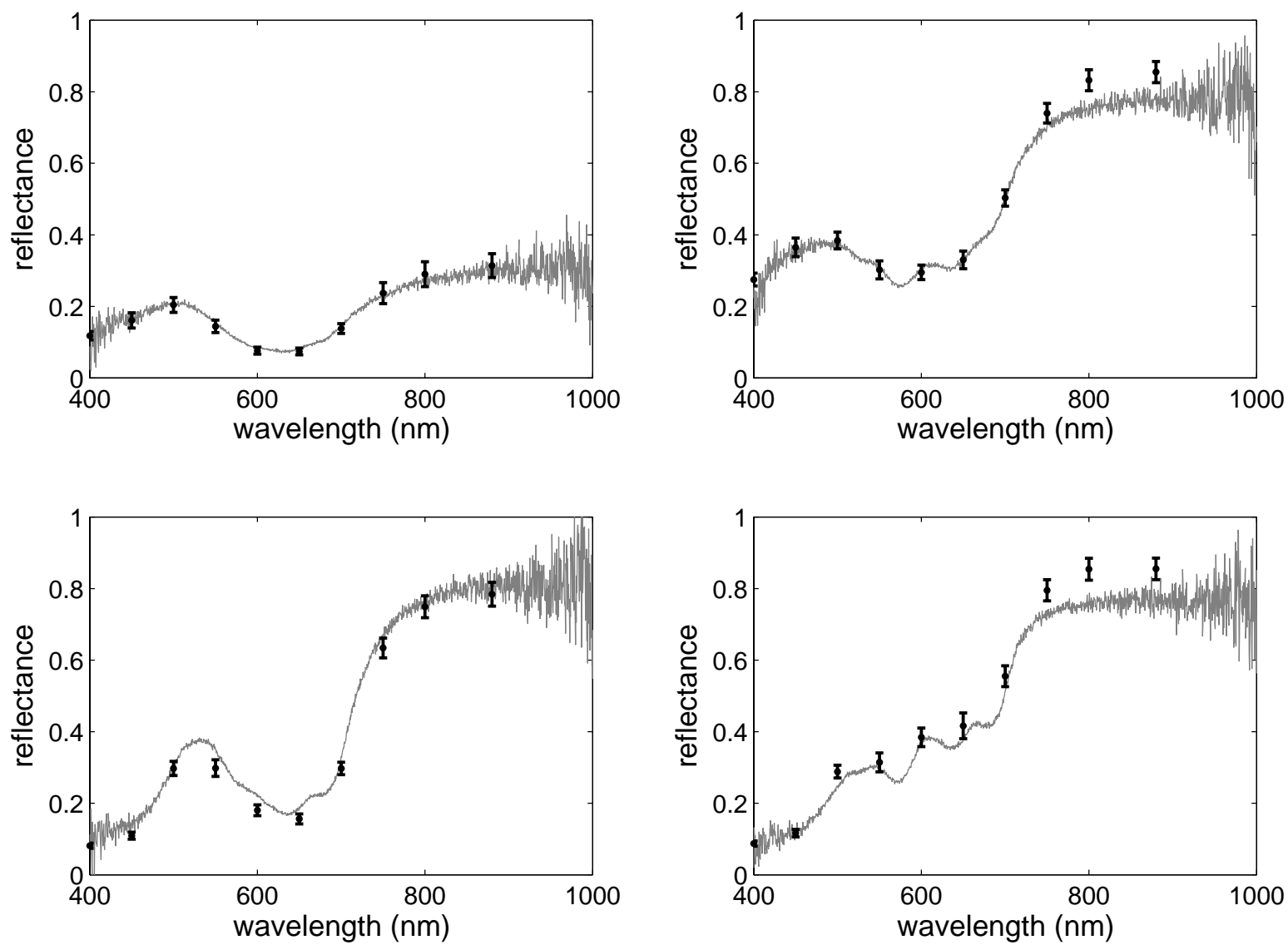

Fig. 7. Spectra of a set of colour patches measured with PRISMS (solid circle with error bars) and those measured with an Ocean Optic HR2000 spectrometer (grey line).

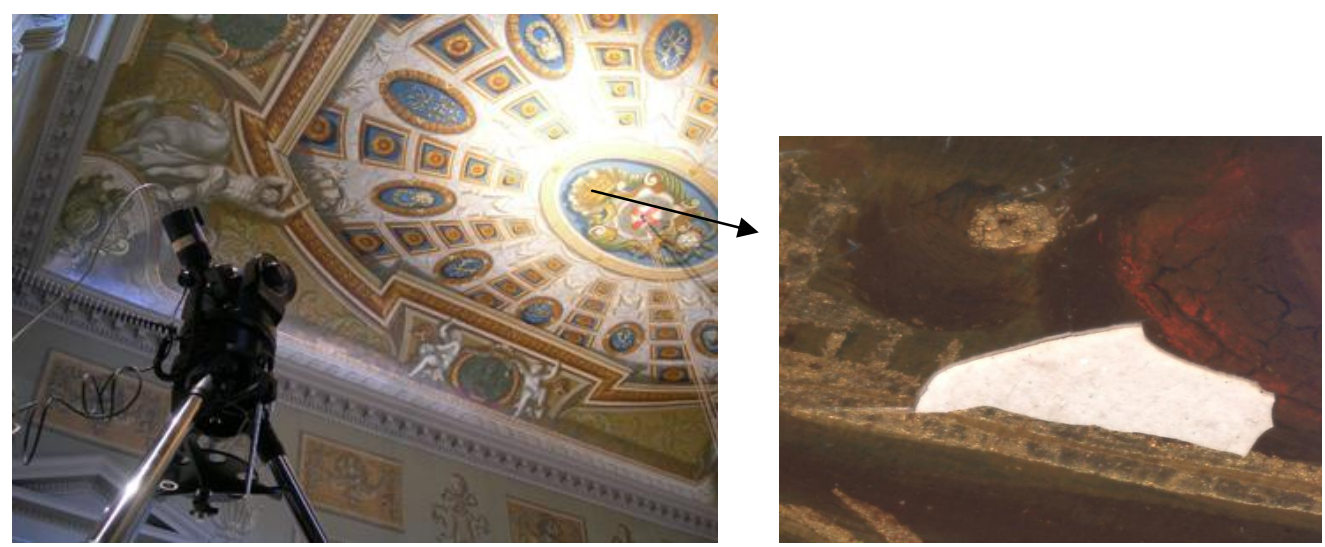

Fig. 8. Left: qualitative remote imaging to inspect the ceiling of the Queen's staircase at Hampton Court Palace; Right: detailed image of paint loss on the ceiling imaged 12-m away. 


\section{ACKNOWLEDGEMENT}

This work is supported by a UK Engineering and Physical Sciences Research Council (EPSRC) grant (EP/E016227/1). We would like to thank Ann Ballantyne, Stuart Burch, Sophie Julien-Lees, Chris Pannell, David Saunders, Jon Ward and QunXi Zhang and for encouragement and support, the Historic Royal Palaces, the British Museum, the Shaan Xi History Museum, the National Gallery, Nottingham Castle Museum and Plumpton church for support, undergraduate project students, Erwan le Gac, Shaun Atherton and Mathieu Cacaud for helping with some of the initial tests and measurements and John Cupitt for maintaining the VIPS/nip software. Tom Vajzovic acknowledges the support of an EPSRC doctoral training scholarship.

\section{REFERENCES}

1 A. Burmester, J. Cupitt, H. Derrien, N. Dessipris, A. Hamber, K. Martinez, M. Müller, D. Saunders, "The Examination of Paintings by Digital Image Analysis", 3rd International Conference on Non Destructive Testing, Microanalytical Methods and Environmental Evaluation for Study and Conservation of Works of Art, Rome, p201, 1993

2 D. Saunders, J. Cupitt, National Gallery Technical Bulletin, 14, 72, 1993.

3 S. Baronti, A. Casini, F. Lotti, S. Porcinai, "Multispectral imaging system for the mapping of pigments in works of art by use of principal-component analysis", Applied Optics, 37, 1299, 1998.

4 C. Balas, V. Papadakis, N. Papadakis, A. Vazgiouraki, G. Themelis, "A Novel hyoer-spectral imaging apparatus for the nondestructive analysis of objects of artistic and historic value", Journal of Cultural Heritage, 4 (1), 330, 2003.

5 J. Hardeberg, F. Schmitt, H. Brettel, "Multispectral color image capture using a liquid crystal tunable filter”, Opt. Eng., 41, 2532 2002.

6 J. Mansfield, M. Attas, C. Majzels, C. Collins, E. Cloutis, H. Mantsch,Vibrational Spectroscopy, 28, 59, 2002.

7 H. Liang, D. Saunders, J. Cupitt, ‘A new multispectral imaging system for examining paintings', Journal of Imaging Science \& Technology, 49, 551, 2005.

8 R. Berns, L. Taplin, F. Imai, E. Day, D. Day, “A comparison of small-aperture and image-based spectrophotometry of paintings”, Studies in Conservation, 50, 253, 2005.

9 J. K. Delaney, E. Walmsley, B. H. Berrie, C. F. Fletcher, 'Multispectral imaging of paintings in the infrared to detect and map blue pigments', Sackler NAS Colloquium - Scientific Examination of Art: Modern Techniques in Conservation and Analysis, p120, 2005.

10 D. Saunders, J. Cupitt, C. White, S. Holt, "The Marc II camera and the scanning initiative at the National Gallery", National Gallery Technical Bulletin, 23, 76, 2002.

11 D. Saunders, R. Billinge, J. Cupitt, N. Atkinson, H. Liang, "A new camera for high resolution infrared imaging of works of art", Studies in Conservation, 52, 277, 2006.

12 H. Wallach, Conservation of Ancient Sites on the Silk Road: $2^{\text {nd }}$ International Conference on the Conservation of Grotto Sites, DunHuang, 2004, in press.

13 E. Lange, Imaging the Past: British Museum Occasional Paper 114, 1-12, British Museum Press, 1996.

14 http://www.vitra.org/

15 J. Cupitt, K. Martinez, “VIPS: An Image Processing System for Large Images”, SPIE Vol. 1663, 19, 1996. 